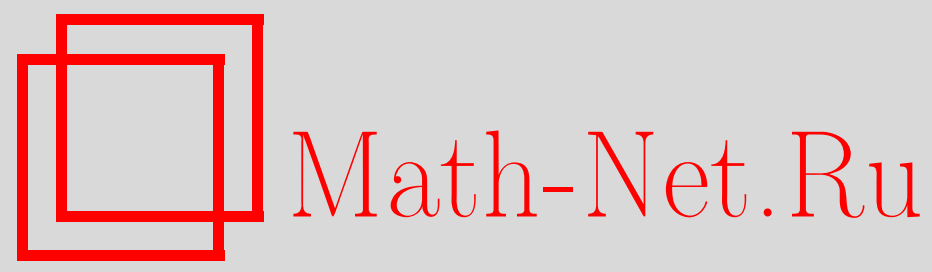

В. А. Добрынский, Об отсутствии циклов у унимодальных отображений квадрата, Матем. заметки, 1998, том 63, выпуск $3,370-378$

DOI: https://doi.org/10.4213/mzm1291

Использование Общероссийского математического портала Math-Net.Ru подразумевает, что вы прочитали и согласны с пользовательским соглашением http://www . mathnet.ru/rus/agreement

Параметры загрузки:

IP: 54.162 .127 .20

26 апреля 2023 г., $17: 40: 54$

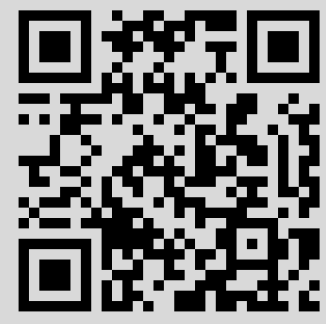




\section{ОБ ОТСУТСТВИИ ЦИКЛОВ \\ У УНИМОДАЛЬНЫХ ОТОБРАЖЕНИЙ КВАДРАТА}

\section{В. А. Добрынский}

Для специального вида унимодальных 2-эндоморфизмов единичного квадрата приведены условия отсутствия у них периодических точек.

Библиограбфия: 4 названия.

Пусть $T$ - некоторое подмножество квадрата $I^{2}=[0,1] \times[0,1]$ и $F$ - отображение $I^{2}$ в себя. Через $\operatorname{Int}(T)$ обозначим внутренность $T$, через $\Gamma(T)=T \backslash \operatorname{Int}(T)$ - его границу, через $\mathrm{NW}(F)$ - множество неблуждающих точек $F$, через $\operatorname{Per}(F)$ - множество периодических точек $F$, через $\|D F\|$ и $\|D \Phi\|$ - якобианы $F$ и $\Phi$, где $\Phi=F(F(F(\cdot)))$. Положим

$$
F^{n}=\underbrace{F(F(\cdots F(\cdot) \cdots))}_{n \text { раз }} .
$$

ОПредЕЛЕниЕ. Отображение $(x, y) \mapsto(\Psi(x, y), x)$ квадрата $I^{2}$ в себя называется унимодальным, если $\Psi(x, y)-C^{1}$-гладкая функция такая, что $\Psi(x, 0)=\Psi(x, 1)=$ $\Psi(0, y)=\Psi(1, y)=0$, и существует точка $(\bar{x}, \bar{y}): \Psi(\bar{x}, \bar{y})>\Psi(x, y)>0$ для любой точки $(x, y) \in \operatorname{Int}\left(I^{2}\right)$ и $(\bar{x}, \bar{y})$ - единственная точка, в которой частные производные $\Psi_{x}^{\prime}$ и $\Psi_{y}^{\prime}$ одновременно обращаются в нуль [1], [2].

Ниже рассматривается семейство унимодальных отображений вида

$$
F:(x, y) \mapsto\left(\lambda f(x)(g(y))^{s}, x\right)
$$

Здесь $\lambda$ и $s,-$ параметры, $0<\lambda \leqslant 1, s>0 ; f(t)-C^{1}$-гладкая унимодальная на $[0,1]$ функция такая, что $f(0)=f(1)=0, f(q)=1, q$ - точка экстремума $f(t),(t-q) f^{\prime}(t)<0$ на $[0,1] \backslash\{q\}\left(f^{\prime}(t)\right.$ - производная от $\left.f(t)\right)$; а $g(t)=h(f(t))$, где $h(t)-C^{1}$-диффеоморфизм $[0,1]$ такой, что $h(0)=0$ и $h^{\prime}(t)>0$ на $[0,1]$.

В [2] доказано, что в пространстве параметров $\lambda$ и $s$ существуют области таких их значений, что отображения вида (1) имеют бесконечно много периодических точек. Есть там также и оценка их числа. Настоящая работа дополняет [1], [2] в том смысле, что здесь указаны области значений параметров, при которых эти отображения не имеют (отличных от неподвижных) периодических точек. 
ОПреДЕЛЕниЕ. Замыкание множества $\left\{(x, y) \in \operatorname{Int}\left(I^{2}\right):\|D F\|=0\right\}$ назьвается критическим множеством $K=K(F)$ отображения $F$.

Данное определение критического множества отличается от [3], но они связаны между собой. Вьпишем в явном виде $K(F)$ и его первые два образа и прообраза:

$$
\begin{gathered}
K=\left\{(x, y) \in I^{2}: y=q, 0 \leqslant x \leqslant 1\right\}, \quad F(K)=\left\{(x, y) \in I^{2}: x=\lambda f(y), 0 \leqslant y \leqslant 1\right\} \\
F^{-1}(K)=\left\{(x, y) \in I^{2}: x=q, 0 \leqslant y \leqslant 1\right\} \\
F^{2}(K)=\left\{(x, y) \in I^{2}: x=\lambda f(y)\left(h\left(\frac{y}{\lambda}\right)\right)^{s}, 0 \leqslant y \leqslant \lambda\right\} \\
F^{-2}(K)=\left\{(x, y) \in I^{2}: q=\lambda f(x)(h(f(y)))^{s}\right\} .
\end{gathered}
$$

Отметим, что $F^{-2}(K)$ существует при $\lambda>q$.

Обозначим $R=F^{2}\left(I^{2}\right)$. Несложно показать, что $R=\left\{(x, y) \in I^{2}: \lambda f(y)(h(y / \lambda))^{s} \leqslant\right.$ $x \leqslant \lambda f(y), 0 \leqslant y \leqslant \lambda\}$. Так чтопри $h(t)=t R=\left\{(x, y) \in I^{2}: \lambda^{1-s} y^{s} f(y) \leqslant x \leqslant \lambda f(y)\right.$, $0 \leqslant y \leqslant \lambda\}$.

Введем обозначения: $M_{1}=\{(x, y): 0 \leqslant x \leqslant q, 0 \leqslant y \leqslant q\}, M_{2}=\{(x, y): q \leqslant x \leqslant 1$, $0 \leqslant y \leqslant q\}, M_{3}=\{(x, y): q \leqslant x \leqslant 1, q \leqslant y \leqslant 1\}, M_{4}=\{(x, y): 0 \leqslant x \leqslant q, q \leqslant y \leqslant 1\}$.

Вычислением проверяется, что справедливы следуюшие соотношения:

$$
F\left(M_{1}\right)=F\left(M_{4}\right) \subset M_{1} \cup M_{2}, \quad F\left(M_{2}\right)=F\left(M_{3}\right) \subset M_{3} \cup M_{4}, \quad F^{2}\left(M_{i}\right)=R, \quad i=\overline{1,4} .
$$

ЛЕмма 1. Если $\lambda \leqslant q$, mo $\mathrm{NW}(F) \subset M_{1}$.

Пусть $\lambda^{*}$ - минимальное решение уравнения $t f(t)=q$ такое, что $\lambda^{*}>q$.

Лемма 2. Пусть $\lambda \in\left(q, \lambda^{*}\right) u s>0$ таковы, что $R \cap \operatorname{Int}\left(M_{4}\right)=\varnothing$. Тогда $F\left(R \cap M_{3}\right) \subset R \cap M_{3} u \mathrm{NW}(F) \subset M_{1} \cup M_{3}$.

ЗАмЕЧАнИЕ. Если $h(t)=t$, то для всех $\lambda: q<\lambda<\lambda^{*}$, можно выбрать одно и то же $s: R \cap \operatorname{Int}\left(M_{4}\right)=\varnothing$. Обозначим его $s^{*}$. Нетрудно проверить, что в качестве $s^{*}$ можно взять 1 . При этом формулировка леммы упрощается.

СлЕДСТВИЕ 1. Если $\lambda<\lambda^{*} u s<s^{*}, \operatorname{mo} F\left(R \cap M_{3}\right) \subset R \cap M_{3} u \mathrm{NW}(F) \subset M_{1} \cup M_{3}$.

Доказательство лемм 1, 2 не представляет затруднений и здесь не приводится.

Лемма 3. Для любых $s: 0<s \leqslant 1$, система уравнений

$$
z_{1}=\lambda f\left(z_{2}\right)\left(f\left(z_{1}\right)\right)^{s}, \quad z_{2}=\lambda f\left(z_{1}\right)\left(f\left(z_{2}\right)\right)^{s}
$$

на отрезке $[0, q]$ имеет только такие решения, что $z_{1}=z_{2}$.

ДокАЗАтЕЛЬСтво. Очевидно, что если $z_{1}=0$, то и $z_{2}=0$, и наоборот. Поэтому, не теряя общности, можно считать, что $z_{i}>0, i=1,2$.

Предположим, что лемма неверна, и существует решение (2) такое, что $z_{1} \neq z_{2}$. Пусть для определенности $z_{2}>z_{1}$. С учетом (2) из данного неравенства вытекает соотношение $f\left(z_{1}\right)\left(f\left(z_{2}\right)\right)^{s}>f\left(z_{2}\right)\left(f\left(z_{1}\right)\right)^{s}$, которое может быть преобразовано к виду $\left(f\left(z_{1}\right)\right)^{1-s}>\left(f\left(z_{2}\right)\right)^{1-s}$. Так как $1-s \geqslant 0$ и $f(t)$ - монотонно возрастающая на [0, $q$ ] функция, последнее означает, что $z_{1}>z_{2}$. 
ЛЕмма 4. Для любых натуральных $N \geqslant 3$ система уравнений

$$
\begin{gathered}
z_{1}=\lambda f\left(z_{N}\right)\left(f\left(z_{N-1}\right)\right)^{s}, \quad z_{2}=\lambda f\left(z_{1}\right)\left(f\left(z_{N}\right)\right)^{s}, \\
z_{i+2}=\lambda f\left(z_{i+1}\right)\left(f\left(z_{i}\right)\right)^{s}, \quad i=\overline{1, N-2},
\end{gathered}
$$

на отрезке $[0, q]$ имеет только такие решения, что $z_{1}=z_{2}=\cdots=z_{N}$.

ДокАЗАТЕЛЬСтво. Очевидно, что если $z_{k}=0$ для некоторого $k$, то $z_{i}=0$ для всех $i \neq k$. Поэтому, не теряя общности, можно считать, что $z_{i}>0, i=\overline{1, N}$.

Предположим, что лемма неверна и существует решение (3) такое, что $z_{k} \neq z_{i}$ для некоторых $k, i: k \neq i$. Зафиксируем индексы $k=\min$ и $i=\max$ так, чтобы переменные $z_{\min }, z_{\max }$ принимали минимальное и максимальноезначения и при этом $z_{\min }<z_{\min }+1$, $z_{\max }>z_{\max +1}$.

Если $0<s \leqslant 1$, рассмотрим следующую пару уравнений:

$$
z_{\max }=\lambda f\left(z_{\max -1}\right)\left(f\left(z_{\max -2}\right)\right)^{s}, \quad z_{\max +1}=\lambda f\left(z_{\max }\right)\left(f\left(z_{\max -1}\right)\right)^{s} .
$$

Поскольку $z_{\max }>z_{\max }+1$, учитьвая (4), получаем неравенство

$$
f\left(z_{\max -1}\right)\left(f\left(z_{\max -2}\right)\right)^{s}>f\left(z_{\max }\right)\left(f\left(z_{\max -1}\right)\right)^{s} .
$$

Преобразуя (5) к виду

$$
\left(f\left(z_{\max -1}\right)\right)^{1-s}>\frac{f\left(z_{\max }\right)}{\left(f\left(z_{\max -2}\right)\right)^{s}}
$$

и учитьвая, что $f\left(z_{\max }\right)>f\left(z_{\max }-2\right)$, окончательно получаем неравенство

$$
\left(f\left(z_{\max -1}\right)\right)^{1-s}>\left(f\left(z_{\max -2}\right)\right)^{1-s} .
$$

Так как $1-s \geqslant 0$ и $f(t)$ - монотонно возрастающая на $[0, q]$ функция, это означает, что $z_{\max -1}>z_{\max }-2$. При этом получается, что справедливо соотношение

$$
f\left(z_{\max -1}\right)\left(f\left(z_{\max -2}\right)\right)^{s}<f\left(z_{\max }\right)\left(f\left(z_{\max -1}\right)\right)^{s},
$$

т.е. $z_{\max }<z_{\max +1}$, что противоречит предположению.

Если $s \geqslant 1$, рассмотрим другую пару уравнений, а именно:

$$
z_{\min }=\lambda f\left(z_{\min -1}\right)\left(f\left(z_{\min -2}\right)\right)^{s}, \quad z_{\min +1}=\lambda f\left(z_{\min }\right)\left(f\left(z_{\min -1}\right)\right)^{s} .
$$

Поскольку $z_{\min }<z_{\min +1}$, из (6) вытекает неравенство

$$
f\left(z_{\min -1}\right)\left(f\left(z_{\min -2}\right)\right)^{s}<f\left(z_{\min }\right)\left(f\left(z_{\min -1}\right)\right)^{s} .
$$

Преобразуя (7) к виду

$$
\left(f\left(z_{\min -1}\right)\right)^{s-1}>\frac{\left(f\left(z_{\min -2}\right)\right)^{s}}{f\left(z_{\min }\right)}
$$

и учитьвая, что $f\left(z_{\min }\right)<f\left(z_{\min }-2\right)$, окончательно получаем неравенство

$$
\left(f\left(z_{\min -2}\right)\right)^{s-1}>\left(f\left(z_{\min -1}\right)\right)^{s-1} .
$$

Так как $s-1 \geqslant 0$ и $f(t)$ - монотонно возрастающая на $[0, q]$ функция, это означает, что $z_{\min -2}>z_{\min }-1$. При этом получается, что справедливо соотношение

$$
f\left(z_{\min }\right)\left(f\left(z_{\min -1}\right)\right)^{s}<f\left(z_{\min -1}\right)\left(f\left(z_{\min -2}\right)\right)^{s},
$$

т.е. $z_{\min }>z_{\min +1}$, что также противоречит предположению. 
ЗАмЕчАниЕ. Аналогично лемме 4 можно доказать следуюшие две леммы.

ЛЕмма 5. Если $f(t) / h(f(t))$ - монотонно возрастающая на $[0, q]$ функиия, то для произвольного натурального $N \geqslant 3$ us: $0<s \leqslant 1$, система уравнений

$$
\begin{gathered}
z_{1}=\lambda f\left(z_{N}\right)\left(h\left(f\left(z_{N-1}\right)\right)\right)^{s}, \quad z_{2}=\lambda f\left(z_{1}\right)\left(h\left(f\left(z_{N}\right)\right)\right)^{s} \\
z_{i+2}=\lambda f\left(z_{i+1}\right)\left(h\left(f\left(z_{i}\right)\right)\right)^{s}, \quad i=\overline{1, N-2}
\end{gathered}
$$

на отрезке $[0, q]$ имеет только такие решения, что $z_{1}=z_{2}=\cdots=z_{N}$.

ЛЕмма 6. Если $h(f(t)) / f(t)$ - монотонно возрастающая на $[0, q]$ функция, то для произвольного натурального $N \geqslant 3 u s \geqslant 1$ система уравнений (8) на отрезке $[0, q]$ имеет только такие решения, что $z_{1}=z_{2}=\cdots=z_{N}$.

Доказательство леммы 5 аналогично доказательству леммы 4 в случае $0<s \leqslant 1$, а леммы $6-$ в случае $s \geqslant 1$.

Прямым следствием утверждений, содержашихся в леммах 3 и 4 , является теорема об отсутствии периодических точек у $F$ при $\lambda \leqslant q$.

Ниже (в теоремах 1-3) предполагается, что $h(t)=t$.

ТеОрема 1. При $\lambda \leqslant q$ отображсние $F$ не имеет периодических точек

а) периода 2 , если $s \leqslant 1$;

б) периода $N$, если $N \geqslant 3$.

ДокАЗАТЕЛЬСтво. Предположим, что $P$ - периодическая точка периода 2 , т.е. $F^{2}(P)=P$. Тогда координаты $P$ и ее итераций удовлетворяют системе уравнений $(2)$. Но согласно лемме 3 при $0<s \leqslant 1$ на $[0, q]$ система (2) имеет только такие решения, что $z_{1}=z_{2}$. Следовательно, точки $P$ и $F(P)$ совпадают, т.е. $P$ - неподвижная точка отображения.

Рассмотрим общий случай. Пусть $N \geqslant 3$ - период точки $P$, так что $F^{N}(P)=P$. Тогда координаты $P$ и ее итераций удовлетворяют системе уравнений (3). Но согласно лемме 4 для любого натурального $N \geqslant 3$ система (3) имеет на отрезке $[0, q]$ только такие решения, что $z_{1}=z_{2}=\cdots=z_{N}$. Значит, $P$ и $F(P)$ совпадают, т.е. $P$ - неподвижная точка отображения.

Пусть $P=(x, y)$ - периодическая периода 2 точка $F$, так что $F^{2}(P)=P$. В переменных $x, y$ это соотношение приобретает вид:

$$
x=\lambda f(y)(f(x))^{s}, \quad y=\lambda f(x)(f(y))^{s} .
$$

Исключая $\lambda$, получаем уравнение семейства кривых, на которых (если существуют) располагаются периодические точки периода 2 отображения:

$$
y(f(y))^{1-s}=x(f(x))^{1-s} .
$$

Одно из решений $(9)$ - диагональ $x=y$. Это решение единственное, если функция $t(f(t))^{1-s}$ монотонно возрастает на $(0,1)$. Последнее вьполняется, в частности, когда $s=1$, или если функция $f(t)+(1-s) t f^{\prime}(t)$ положительна на $(0,1)$.

Таким образом, имеет место 
ТЕорема 2. Если при некотором $s>0$ функиия $f(t)+(1-s) t f^{\prime}(t)$ положительна на $(0,1)$, то при этом $s$ отображение $F$ не имеет периодических точек периода 2.

ДокАЗАТЕЛЬСТво. Положительность $f(t)+(1-s) t f^{\prime}(t)$ влечет за собой положительность производной от функции $t(f(t))^{1-s}$ и, как следствие, монотонное возрастание самой функции.

В частном случае (при $s=1$ ) можно доказать, что у $F$ нет циклов периода 3 при значениях $\lambda$ много больших $q$. Именно, справедлива следующая теорема.

Теорема 3. Пусть $s=1 u \lambda<\bar{q}$, где $\bar{q}-$ ближайшая $\kappa 0$ точка әкстремума функиии $t f(t)$. Тогда отображение $F$ не имеет периодических точек периода 3.

ДокАЗАТЕЛЬСтво. В самом деле, как нетрудно проверить, координаты 3-периодических точек удовлетворяют системе уравнений

$$
\lambda^{1-s} y^{s} f(y)=x(f(x))^{s^{2}}, \quad \lambda^{1-s} x^{s} f(x)=z(f(z))^{s^{2}}
$$

где $x, y$ - координаты точки $P: P=F^{3}(P)$, а $z=\lambda f(x)(f(y))^{s}$. При $s=1(10)$ превращается в систему уравнений $x f(x)=y f(y)=z f(z)$. Обозначим через $\bar{q}$ ближайшую к 0 точку экстремума функции $t f(t)$. Очевидно, $\bar{q}>q$. В силу монотонности $t f(t)$ на $[0, \bar{q}]$ всякое решение последней системы таково, что $x=y=z$. Следовательно, $P$ - неподвижная точка $F$.

ЛЕмма 7. Если $\lambda<1$ us $>0$ maкие, что $R \cap M_{4}=\varnothing, \operatorname{mo} \Phi\left(R \cap M_{3}\right) \subset \operatorname{Int}\left(R \cap M_{3}\right)$.

ДокАЗАТЕЛЬСтво. Поскольку условия леммы совпадают с условиями леммы 2 , то $F\left(R \cap M_{3}\right) \subset R \cap M_{3}$. А так как $F$ - диффеоморфизм $R \cap M_{3}$ на $F\left(R \cap M_{3}\right)$, то $\Phi-$ диффеоморфизм $R \cap M_{3}$ на $\Phi\left(R \cap M_{3}\right)$ и $\Phi\left(R \cap M_{3}\right) \subset R \cap M_{3}$. Таким образом, если показать, что гранишы множеств $R \cap M_{3}$ и $\Phi\left(R \cap M_{3}\right)$ не пересекаются, то лемма будет доказана. Заметим теперь, что граница множества $R \cap M_{3}$ образована подмножествами множеств $K, F(K)$ и $F^{2}(K)$ и выполняются соотношения

$$
\begin{gathered}
F\left(K \cap R \cap M_{3}\right) \subset F(K) \cap R \cap M_{3}, \\
F\left(F(K) \cap R \cap M_{3}\right) \subset F^{2}(K) \cap R \cap M_{3}, \\
F\left(F(K) \cap R \cap M_{3}\right) \cap\left(F(K) \cap R \cap M_{3}\right)=\varnothing, \\
F\left(F^{2}(K) \cap R \cap M_{3}\right) \cap\left(F^{2}(K) \cap R \cap M_{3}\right)=\varnothing, \\
F\left(K \cap R \cap M_{3}\right) \cap\left(K \cap R \cap M_{3}\right)=\varnothing .
\end{gathered}
$$

Справедливость соотношений (11), (12) устанавливается непосредственно вычислениями. Далее, условие $R \cap M_{4}=\varnothing$ означает по сути, что $R$ не пересекается с подмножеством $F^{-1}(K) \cap\{(x, y): y>q\}$ прообраза $F^{-1}(K)$. Но это значит, что $F$-образ множества $F^{2}(K) \cap R \cap M_{3}$ не имеет точек пересечения с множеством $K \cap R \cap M_{3}$. Следовательно, $\Phi\left(K \cap R \cap M_{3}\right) \cap K \cap R \cap M_{3}=\varnothing$. Одновременно выполняются соотношения: $\Phi\left(K \cap R \cap M_{3}\right) \cap F(K) \cap R \cap M_{3}=\varnothing, \Phi\left(K \cap R \cap M_{3}\right) \cap F^{2}(K) \cap R \cap M_{3}=\varnothing$. В самом деле, в противном случае существует точка $P$ такая, что $\Phi(P)$ принадлежит $F(K) \cap R \cap M_{3}$ или $F^{2}(K) \cap R \cap M_{3}$. Это означает, что одна из точек $F^{2}(P)=F^{-1}(\Phi(P))$ или $F(P)=F^{-2}(\Phi(P))$ принадлежит $K \cap R \cap M_{3}$. Но это противоречит (12). Таким образом, $\Phi\left(K \cap R \cap M_{3}\right) \subset \operatorname{Int}\left(R \cap M_{3}\right)$. 
Аналогично устанавливается справедливость соотношений $\Phi\left(F(K) \cap R \cap M_{3}\right) \subset$ $\operatorname{Int}\left(R \cap M_{3}\right)$ и $\Phi\left(F^{2}(K) \cap R \cap M_{3}\right) \subset \operatorname{Int}\left(R \cap M_{3}\right)$. Отсюда вытекает, что граница множества $\Phi\left(R \cap M_{3}\right)$ лежит внутри $R \cap M_{3}$.

Отметим, что внешняя сложность формулировки условия леммы обманчива. На самом деле все сводится к проверке условия $\lambda f(y)(h(y / \lambda))^{s}>q$ при $y \in[q, \lambda]$, поскольку $F^{2}(K) \cap R \cap M_{3}$ - ближайшая к $M_{4}$ граница множества $R \cap M_{3}$ - является графиком функции $x=\lambda f(y)(h(y / \lambda))^{s}$. Таким образом, имеет место следующая

Лемма 8. Если $\lambda: q<\lambda<\lambda^{*}, u s>0$ такие, что на $[q, \lambda]$ выполняется соотношение $\lambda f(y)(h(y / \lambda))^{s}>q$, mо $\Phi\left(R \cap M_{3}\right) \subset \operatorname{Int}\left(R \cap M_{3}\right)$.

При $h(t)=t$ можно указать единое $s^{*}=1$ такое, что для любых $\lambda$ и $s\left(q<\lambda<\lambda^{*}\right.$ и $0<s<1)$, на $[q, \lambda] \lambda f(y)(h(y / \lambda))^{s}>q$. Это позволяет упростить формулировку леммы 8 следующим образом.

СлЕДСтвиЕ 2. Ecлu $h(t)=t, \lambda \in\left(q, \lambda^{*}\right) u s \in(0,1), \operatorname{mo} \Phi\left(R \cap M_{3}\right) \subset \operatorname{Int}\left(R \cap M_{3}\right)$.

Отметим, что условие $R \cap M_{3} \cap M_{4}=\varnothing$ существенно и не может быть ослаблено, скажем, до $\operatorname{Int}\left(R \cap M_{3}\right) \cap M_{4}=\varnothing$ или $\operatorname{Int}\left(M_{4}\right) \cap\left(R \cap M_{3}\right)=\varnothing$. Tо же самое касается формулировок леммы 8 и следствия 2 , для которых нарушение условий $\lambda<\lambda^{*}$, $s<1$ (даже до $\lambda=\lambda^{*}, s=1$ ) может привести к тому, что сформулированные вьше утверждения уже не будут вьполняться.

Рассмотрим, например, отображение $(x, y) \mapsto(\lambda f(x) f(y), x)$. Здесь $h(t)=t, s=1$. Если $\lambda=\lambda^{*}$, то $\lambda^{*} f\left(\lambda^{*}\right)=q$, и в $M_{3}$ отображение $F$ имеет цикл периодических периода 3 точек: $(q, q) \mapsto\left(\lambda^{*}, q\right) \mapsto\left(q, \lambda^{*}\right) \mapsto(q, q)$. Ясно, что в этом случае утверждения лемм 7,8 и следствия 2 не выполняются.

Изложенные вьше результаты могут быть усилены, если функция $\widetilde{T}=\lambda f(t)(h(t / \lambda))^{s}$ унимодальна на $[q, \lambda]$. Это условие порождает ограничения на свойства функций $f(t)$ и $h(t)$.

Обозначим через $\lambda_{*}$ тот корень уравнения $f\left(\lambda_{*}\right)+\lambda_{*} f^{\prime}\left(\lambda_{*}\right)=0$, который больше чем $q$, и меньше чем $\lambda_{*}$.

ТЕОрема 4. Если $f^{\prime \prime}(t)<0, h^{\prime \prime}(t)<0$ на $\left[q, \lambda_{*}\right] u$, кроме того, выполняется одно из условий:

a) $\left(R \cap M_{3}\right) \cap M_{4}=\varnothing$ или

б) $\lambda f(y)(h(y / \lambda))^{s}>q$ для $y \in[q, \lambda]$,

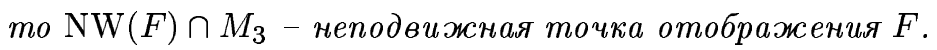

При $h(t)=t$ условие теоремы 4 можно упростить следующим образом.

Tеорема 5. Ecлu $f^{\prime \prime}(t)<0$ на $[q, 1], \lambda \in\left(q, \lambda_{*}\right) u s \in(0,1)$, mo $\mathrm{NW}(F) \cap M_{3}-$ неподвижная точка отобрахсения $F$.

ДокАЗАТЕЛЬСТво ТЕОРЕмы 4. Пусть $\rho(T, V)$ - расстояние между замкнутьми множествами $T$ и $V$, т.е. $\rho(T, V)=\min _{t, v} \rho(t, v)$, где $\rho(t, v)$ - евклидово расстояние между точками $t \in T$ и $v \in V$. Так как $\left(R \cap M_{3}\right) \cap M_{4}=\varnothing$, то $\rho\left(R \cap M_{3}, M_{4}\right)=\gamma_{0}>0$. Согласно предположению $x=\lambda f(y)(h(y / \lambda))^{s}-$ вьпуклая и унимодальная на $[q, 1]$ функция. Следовательно, на $[q, \lambda]$ минимальное значение, равное $q+\gamma_{0}$, она достигает в одном из концов отрезка. Поэтому $q+\gamma_{0}=\min \left\{\lambda(h(q / \lambda))^{s}, \lambda f(\lambda)\right\}$. 


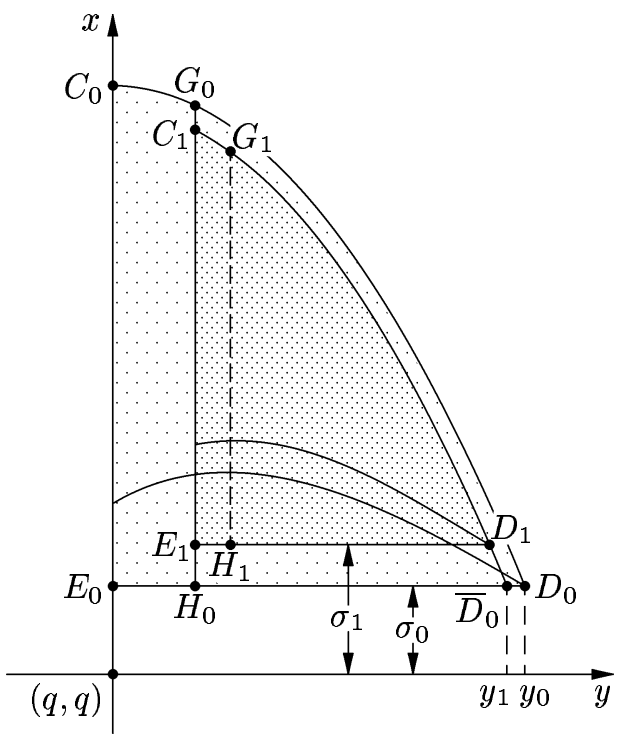

Рис. 1

Рассмотрим в $M_{3}$ криволинейньй треугольник $C_{0} D_{0} E_{0}$, образованньй отрезками $\left\{(x, q): q+\gamma_{0} \leqslant x \leqslant \lambda\right\},\left\{\left(q+\gamma_{0}, y\right): q \leqslant y \leqslant y_{0}\right\}$ и дугой $\left\{(x, y): x=\lambda f(y), q \leqslant y \leqslant y_{0}\right\}$, где $y_{0}$ - значение координаты $y$ точки $D_{0}$, в которой кривая $x=\lambda f(y)$ пересекает прямую $x=q+\gamma_{0}$, с вершинами $C_{0}=(\lambda, q), D_{0}=\left(q+\gamma_{0}, y_{0}\right)$ и $E_{0}=\left(q+\gamma_{0}, q\right)$. Отметим, что $y_{0}=\lambda$, если $f(\lambda) \leqslant(h(q / \lambda))^{s}$; в противном случае $y_{0}>\lambda$.

По построению $C_{0} D_{0} E_{0} \supset R \cap M_{3}$ и $\rho\left(C_{0} D_{0} E_{0}, M_{4}\right)=\gamma_{0}$. Покажем, что сушествует аналогичный $C_{0} D_{0} E_{0}$ криволинейный треугольник $C_{1} D_{1} E_{1}$ такой, что $C_{1} D_{1} E_{1} \subset$ $\operatorname{Int}\left(C_{0} D_{0} E_{0}\right)$ и $\Phi\left(C_{0} D_{0} E_{0}\right) \subset C_{1} D_{1} E_{1}$.

Рассмотрим $F\left(C_{0} D_{0} E_{0}\right)$. Поскольку $F:\left(q+\gamma_{0}, y\right) \mapsto\left(x, q+\gamma_{0}\right), K \rightarrow F(K), F(K) \rightarrow$ $F^{2}(K)$, то $F\left(C_{0} D_{0} E_{0}\right) \subset C_{0} D_{0} E_{0} \cap\left\{(x, y): y>q+\gamma_{0}\right\}$. Обозначим через $H_{0}$ точку $\left(q+\gamma_{0}, q+\gamma_{0}\right)$, через $G_{0}$ - точку $\left(\lambda f\left(q+\gamma_{0}\right), q+\gamma_{0}\right)$, так что $D_{0} H_{0} G_{0}=C_{0} D_{0} E_{0} \cap\{(x, y)$ : $\left.y \geqslant q+\gamma_{0}\right\}$ и $F\left(C_{0} D_{0} E_{0}\right) \subset D_{0} H_{0} G_{0}$.

Рассмотрим $F\left(D_{0} H_{0} G_{0}\right)$. Так как $F\left(D_{0}\right)=F^{2}(K) \cap H_{0} G_{0}$, а $F\left(E_{0}\right)=G_{0}$, то $F\left(D_{0} H_{0}\right) \subset H_{0} G_{0}$, a $F\left(H_{0} G_{0}\right)=C_{1} \bar{D}_{0}=\left\{(x, y): x=\lambda_{0} f(y), q+\gamma_{0} \leqslant y \leqslant y_{1}\right\}$, где $C_{1}=F\left(H_{0}\right), \lambda_{0}=\lambda\left(h\left(f\left(q+\gamma_{0}\right)\right)\right)^{s}<\lambda$, а $y_{1}-$ значение координаты $y$ точки $\bar{D}_{0}$, в которой пересекаются кривая $x=\lambda_{0} f(y)$ с прямой $x=q+\gamma_{0}$. Заметим, что поскольку $\lambda_{0}<\lambda$, то $y_{1}<y_{0}$. Из сказанного ясно, что $F\left(D_{0} H_{0} G_{0}\right) \subset D_{0} H_{0} G_{0} \cap\left\{(x, y): x=\lambda_{0} f(y)\right.$, $\left.q+\gamma_{0} \leqslant y \leqslant y_{1}\right\}$. Таким образом, $F\left(D_{0} H_{0} G_{0}\right) \subset \bar{D}_{0} H_{0} C_{1}=D_{0} H_{0} G_{0} \cap\{(x, y): x=$ $\left.\lambda_{0} f(y), q+\gamma_{0} \leqslant y \leqslant y_{1}\right\}$.

Рассмотрим $F\left(\bar{D}_{0} H_{0} C_{1}\right)$. Как и вьше, две стороны (из трех) треугольника отображаются в соседние $\left(F\left(\bar{D}_{0} H_{0}\right) \subset H_{0} C_{1}, F\left(H_{0} C_{1}\right) \subset C_{1} \bar{D}_{0}\right)$, а третья сторона - дуга $C_{1} \bar{D}_{0}=\left\{(x, y): x=\lambda_{0} f(y), q+\gamma_{0} \leqslant y \leqslant y_{1}\right\}-$ переходит в дугу $F\left(C_{1} \bar{D}_{0}\right)$ кривой $x=\lambda f\left(\lambda_{0} y / \lambda\right)(h(y / \lambda))^{s}$. Поскольку $\lambda_{0}<\lambda$, для всех $y \in\left[q+\gamma_{0}, y_{1}\right]$ $f(y)<f\left(\lambda_{0} y / \lambda\right)$ и, следовательно, точки данной дуги лежат вьше (строго “над") кривой $x=\lambda f(y)(h(y / \lambda))^{s}$. Это значит, что $\Phi\left(C_{0} D_{0} E_{0}\right) \subset \operatorname{Int}\left(C_{0} D_{0} E_{0}\right)$. Положим $\gamma_{1}=\rho\left\{F\left(C_{1} \bar{D}_{0}\right), M_{4}\right\}$. Согласно проведенным вьше рассуждениям $\gamma_{1}>\gamma_{0}$. Определим треугольник $C_{1} D_{1} E_{1}$ как множество $\left\{(x, y): x \geqslant q+\gamma_{1}, y \geqslant q+\gamma_{0}\right\} \cap \bar{D}_{0} H_{0} C_{1}$, где вершины $D_{1}$ и $E_{1}$ совпадают с точками пересечения прямой $x=q+\gamma_{1}$ с кривой 
$x=\lambda_{0} f(y)$ и прямой $y=q+\gamma_{0}$ соответственно. Очевидно, для построенного таким образом треугольника $C_{1} D_{1} E_{1}$ справедливы сформулированные выше требования, т.е. $C_{1} D_{1} E_{1} \subset \operatorname{Int}\left(C_{0} D_{0} E_{0}\right)$ и $\Phi\left(C_{0} D_{0} E_{0}\right) \subset C_{1} D_{1} E_{1}$.

Применяя изложенные вьше рассуждения к треугольнику $C_{1} D_{1} E_{1}$, можно построить треугольник $C_{2} D_{2} E_{2}$, обладающий теми же свойствами, что и $C_{1} D_{1} E_{1}: C_{2} D_{2} E_{2} \subset$ $\operatorname{Int}\left(C_{1} D_{1} E_{1}\right)$ и $\Phi\left(C_{1} D_{1} E_{1}\right) \subset C_{2} D_{2} E_{2}$, и т.д. Так как

$$
\bigcap_{j=1}^{\infty} F^{j}\left(R \cap M_{3}\right) \subset C_{n} D_{n} E_{n}
$$

для любого натурального $n$, то

$$
\bigcap_{j=1}^{\infty} F^{j}\left(R \cap M_{3}\right) \subset \bigcap_{n=1}^{\infty} C_{n} D_{n} E_{n} \stackrel{\text { def }}{=} \widetilde{C} \widetilde{D} \widetilde{E}
$$

Покажем, что $\widetilde{C} \widetilde{D} \widetilde{E}-$ точка.

Очевидно, что каждая из последовательностей $\left\{E_{n}\right\},\left\{C_{n}\right\},\left\{D_{n}\right\}, n \in \mathbb{Z}_{+}$, точек $E_{n}, C_{n}, D_{n}$ является сходящейся и имеет предел. В самом деле, по построению каждая из точек $E_{n+1}, C_{n+1}, D_{n+1}$ лежит соответственно выше и правее, ниже и правее, выше и левее точек $E_{n}, C_{n}, D_{n}$, так что их координаты образуют монотонно возрастающие (убывающие), ограниченные сверху (снизу) последовательности. Поэтому последовательности $\left\{E_{n}\right\},\left\{C_{n}\right\}$ и $\left\{D_{n}\right\}$ имеют пределы. Обозначим $\widetilde{E}=\lim E_{n}, \widetilde{C}=\lim C_{n}$, $\widetilde{D}=\lim D_{n}$ и рассмотрим последовательности $\left\{H_{n}\right\},\left\{G_{n}\right\}$ и $\left\{\bar{D}_{n}\right\}$. Учитьвая, что $\gamma_{n}$ - ограниченная, монотонно возрастающая последовательность, нетрудно заметить, что $\lim H_{n}=\lim E_{n}=\widetilde{E}$. А так как $H_{n} \in\{(x, y): x=y\}, \widetilde{E}$ также лежит на диагонали. Далее, из соотношений $G_{n}=F\left(E_{n}\right), C_{n}=F\left(H_{n}\right)$ в силу непрерьвности $F$ вытекает, что $\lim G_{n}=\lim C_{n}=\widetilde{C}$ и, кроме того, $F(\widetilde{E})=\widetilde{C}$. Аналогично получается, что $\lim \bar{D}_{n}=\lim D_{n}=\widetilde{D}$ и $F(\widetilde{C})=\widetilde{D}$. Поэтому наборы отрезков $E_{n} C_{n}$ и $D_{n} E_{n}$ образуют сходящиеся последовательности. Их пределами являются точки либо отрезки. Логически допустимы следующие варианты:

a) $\widetilde{E}=\widetilde{C}=\widetilde{D}$

б) $\widetilde{E}=\widetilde{C} \neq \widetilde{D}$ или $\widetilde{E} \neq \widetilde{C}=\widetilde{D}$;

в) $\widetilde{E} \neq \widetilde{C} \neq \widetilde{D}$.

Если вьполнено а), то поскольку $E_{n}, C_{n} D_{n}-$ крайние точки $C_{n} D_{n} E_{n}$, т.е. такие, на которых значения координат точек $C_{n} D_{n} E_{n}$ достигают своих максимальных и минимальных величин, получаем, что $\bigcap_{n=1}^{\infty} C_{n} D_{n} E_{n}$ - точка.

Случай б) в действительности не реализуется. В самом деле, с учетом $F(\widetilde{E})=\widetilde{C}$ и $F(\widetilde{C})=\widetilde{D}$ из $\widetilde{E}=\widetilde{C}$ получаем, что $\widetilde{C}=F(\widetilde{E})=F(\widetilde{C})=\widetilde{D}$, а из $\widetilde{C}=\widetilde{D},-$ что $F(\widetilde{E})=\widetilde{C}=F(\widetilde{C})=F(\widetilde{D})$, т.е. $\widetilde{E}=\widetilde{C}=\widetilde{D}$.

Рассмотрим случай в). Как уже отмечалось, последовательности $\left\{E_{n} C_{n}\right\}$ и $\left\{D_{n} E_{n}\right\}$ сходятся соответственно к отрезкам $\widetilde{E} \widetilde{C}$ и $\widetilde{D} \widetilde{E}$. Покажем, что последовательность дуг $\left\{C_{n} D_{n}\right\}$ также сходится к дуге $\widetilde{C} \widetilde{D}$. В самом деле, по построению для всех $n \in \mathbb{Z}_{+}$дуги $C_{n} D_{n}$ являются графиками функций $x=\lambda_{n-1} f(y)$, где $q<\lambda_{n}<\lambda_{n-1}$, и $C_{n} D_{n} \cap$ $C_{n-1} D_{n-1}=\varnothing$. Таким образом, $C_{n} D_{n}$ образуют сходящуюся последовательность. А так как $0<\left|f^{\prime}(x)\right|<+\infty$ на $C_{1} D_{1} E_{1}$ и $C_{n} D_{n} E_{n} \subset C_{1} D_{1} E_{1}$ для $n \in \mathbb{Z}_{+}$, то сходимость 
равномерная и, значит, $\widetilde{C} \widetilde{D}=\lim C_{n} D_{n}-$ дуга, совпадающая с графиком функции $x=\widetilde{\lambda} f(y)$, где $\widetilde{\lambda}=\lim \lambda_{n}$. Таким образом, $\bigcap_{n=1}^{\infty} C_{n} D_{n} E_{n}-$ точка или треугольник. Последнее, однако, невозможно, так как по построению $\widetilde{E} \in\{(x, y): x=y\}$ и, кроме того, попарно совпадают значения $y$-координат точек $\widetilde{E}$ и $\widetilde{C}$ и $x$-координат точек $\widetilde{E}$ и $\widetilde{D}$. Если обозначить через $(\widetilde{y}, \widetilde{y})$ координаты $\widetilde{E}$, то (ввиду $F(\widetilde{E})=\widetilde{C}$ и $F(\widetilde{C})=\widetilde{D})$ из сказанного вытекает, что $\widetilde{C}=(\widetilde{x}, \widetilde{y})$, где $\widetilde{x}=\widetilde{\lambda} f(\widetilde{y})(h(f(\widetilde{y})))^{s}$ и $\widetilde{x}>\widetilde{y}$, и $\widetilde{D}=(\widetilde{y}, \widetilde{x})$. Следовательно, вьполняется соотношение $\widetilde{y}=\tilde{\lambda} f(\widetilde{x})\left(h(f(\widetilde{y}))^{s}\right.$. В паре с $\widetilde{x}=\widetilde{\lambda} f(\widetilde{y})(h(f(\widetilde{y})))^{s}$ оно образует систему уравнений для определения $\widetilde{x}$ и $\widetilde{y}$, из которой получается, что $\widetilde{x} f(\widetilde{x})=\widetilde{y} f(\widetilde{y})$. Но согласно определению $\lambda_{*}$ на $\left[q, \lambda_{*}\right]$ последнее уравнение имеет единственное решение $\widetilde{x}=\widetilde{y}$. Следовательно, наше предположениеневерно и, значит, $\widetilde{C} \widetilde{D} \widetilde{E}-$ точка.

С учетом теоремы 5 можно расширить область значений $\lambda$ и $s$, при которых $F$ не имеет (отличных от неподвижных) периодических точек.

Теорема 6. Ecлu $f^{\prime \prime}(t)<0$ на $[q, 1], \lambda \in\left(q, \lambda_{*}\right) u s \in(0,1)$, mo $F$ не имеет (отличных от неподвижных) периодических точек.

ДокАЗАтельство. Рассмотрим действие отображения $F$ на $R \cap M_{i}, i=\overline{1,3}$. С одной стороны, поскольку $F: F^{-1}(K) \rightarrow K,(0,0) \mapsto(0,0)$, и $x$-координата любой точки $P$ переводится отображением в $y$-координату ее образа $F(P)$, справедливы соотношения: $F\left(R \cap M_{1}\right) \subset\left(R \cap M_{1}\right) \cup\left(R \cap M_{2}\right)$ и $F\left(R \cap M_{2}\right) \subset R \cap M_{3}$. С другой стороны, согласно лемме $2 F\left(R \cap M_{3}\right) \subset R \cap M_{3}$. Отсюда ясно, что NW $(F) \cap M_{2}=\varnothing$ и $\mathrm{NW}(F)=\left(\mathrm{NW}(F) \cap M_{1}\right) \cup\left(\mathrm{NW}(F) \cap M_{3}\right)$, так что $\operatorname{Per}(F)=\operatorname{Per}\left(\left.F\right|_{M_{1}}\right) \cup \operatorname{Per}\left(\left.F\right|_{M_{3}}\right)$. Но по теореме $5 \operatorname{Per}\left(\left.F\right|_{M_{3}}\right)$ - неподвижная точка $F$. А из лемм 3 и 4 следует, что на $M_{1}$ $F$ не имеет (отличных от неподвижных) периодических точек.

Большинство результатов ранее опубликованы в [4].

\section{СПИСОК ЦИТИРОВАННОЙ ЛИТЕРАТУРЫ}

[1] Добрынский В.А. Критические множества и унимодальные отображения квадрата // Докл. РАН. 1995. Т. 341. № 4. С. 442-445.

[2] Добрынский В.А. Критические множества и унимодальные отображения квадрата // Матем. заметки. 1995. Т. 58. № 5. С. 669-680.

[3] Gumowski I., Mira C. Dynamique Chaotique. Transformations Ponctuelles. Transition Ordre-Desordre. Toulouse: Editions Cepadues, 1980.

[4] Добрынский В. А. Свойства циклов отображений, порождаемых разностными уравнениями второго порядка с нелинейностью типа произведение. Препринт. Киев: ИМ НАН Украины, 1994. 